\title{
Introducing the theory of relativity
}

\author{
Peter G. Nelson ${ }^{1}$
}

Published online: 17 December 2015

(C) The Author(s) 2015. This article is published with open access at Springerlink.com

\begin{abstract}
A simple way of introducing the theory of relativity to chemistry students is presented. This is based on experimental observations of the variation in the mass of an electron with speed. Analysis of these generates the equations chemists use, and provides a basis for critical discussion of the theory.
\end{abstract}

Keywords Mass $\cdot$ Speed $\cdot$ Energy $\cdot$ Light $\cdot$ Relativity

\section{Introduction}

The theory of relativity enters into chemistry in two main ways. First, it helps to explain the chemistry of heavy elements (Banna 1985; Norrby 1991; Balasubramanian 1997; Thayer 2005). A good example is the inert pair effect (Grimm and Sommerfeld 1926; Sidgwick 1927). This concerns the formation of compounds by heavy main-group elements with a valency that is two less than the group valency (e.g. $\mathrm{TlCl}$ and $\mathrm{PbCl}_{2}$ ). Thermodynamic analysis shows that these compounds are formed because the energies of outer $\mathrm{s}$ electrons do not decrease down a group to the same extent as those of other electrons (Hopkins and Nelson 1984). This is attributable to the high speeds attained by electrons moving near a nucleus with a high nuclear charge, causing them to increase in mass and slow down. This affects s electrons more than other electrons because they have a higher probability of being near the nucleus. The slowing down means that they spend longer in the region where nuclear attraction is high, which raises their binding energy relative to other electrons and lowers their chemical activity.

Secondly, the theory of relativity explains the difference between the mass of an atom and the sum of the masses of the particles of which it is composed (Atwood and Sheline

Peter G. Nelson

p.g.nelson@hull.ac.uk

1 Department of Chemistry, University of Hull, Hull HU6 7RX, UK 
1989). For example, the mass of a ${ }^{12} \mathrm{C}$ atom is (by definition) exactly $12 \mathrm{u}$, compared with a mass of about $12.09894 \mathrm{u}$ for six protons, six neutrons, and six electrons (Cohen et al. 2007). ${ }^{1}$

In chemistry courses, the results of the theory of relativity are usually assumed. To explain the above phenomena, the equations

$$
\begin{gathered}
m=m_{0}\left(1-v^{2} / c^{2}\right)^{-1 / 2} \\
E=m c^{2}
\end{gathered}
$$

are taken as starting points ( $m=$ mass, $m_{0}=$ rest mass, $v=$ speed, $c=$ speed of light, $E=$ energy). This spares students from having to grapple with the derivation of these equations, but means that they have to take them on trust. Here I would like to suggest a more satisfying approach.

\section{Variation of mass with speed}

The approach begins by returning to an experiment used to find the ratio of the mass $(m)$ to the charge $(q)$ of an electron (Nelson 2002). This involves passing cathode rays through a uniform electric field $(E)$, measuring the radius $(r)$ of the circular path they take, and then using a uniform magnetic field $(B)$ at right angles to annul the deflection of the beam. Under the first conditions, the electric force on each electron $(E q)$ is equal to the particle's rate of change of momentum $\left(m v^{2} / r\right)$; under the second, the electric force is balanced by the magnetic force $(B v q)$. The second relation gives

$$
v=E / B
$$

From this and the first relation

$$
m / q=B^{2} r / E
$$

Experiments with cathode rays of low energy give $m / q=-5.69 \times 10^{-9} \mathrm{~g} \mathrm{C}^{-1}$. Combining this with the charge on an electron $\left(q=-1.602 \times 10^{-19} \mathrm{C}\right)$ gives $m=9.11 \times 10^{-28} \mathrm{~g}$.

However, when experiments like this are repeated with cathode rays of high energy, the values of $m$ are found to increase with speed (calculated from Eq. 3). This was investigated by Kaufmann (1901) before Einstein put forward his theory of relativity (see Miller 1997). His results, along with some of those obtained later by Bucherer (1909), are plotted in Fig. 1 (Shankland 1960: Chap. 2, Sect. 4). These show that $m$ rises increasingly rapidly with $v$, and apparently approaches a limit equal to the speed of light $\left(c=2.998 \times 10^{8} \mathrm{~m} \mathrm{~s}^{-1}\right)$. The limits $\left(m=m_{0}\right.$ at $v=0, m=\infty$ at $\left.v=c\right)$ suggest changing the variables to $\alpha=m / m_{0}$ and $\beta=v / c$. The variable $\alpha$ then varies from one to infinity as $\beta$ varies from zero to one.

A simple function that varies between these limits is $\alpha_{1}=(1-\beta)^{-1}$. This does not fit the data (Table 1). Somewhat better functions are $\alpha_{2}=\left(1-\beta^{2}\right)^{-1}$ and $\alpha_{3}=(1-\beta)^{-1 / 2}$. Better still is the function $\alpha_{4}=\left(1-\beta^{2}\right)^{-1 / 2}$, corresponding to Eq. 1. More precise measurements indicate that Eq. 1 holds to $\pm 0.05 \%$ in the range $\beta=0.2-0.5$ (Guye and Lavanchy 1915) and $\pm 0.5 \%$ in the range $\beta=0.6-0.8$ (Rogers et al. 1940).

\footnotetext{
1 The unit $\mathrm{u}$ is the unified atomic mass unit or dalton, Da.
} 
Fig. 1 Plot of electron mass against speed (Shankland 1960)

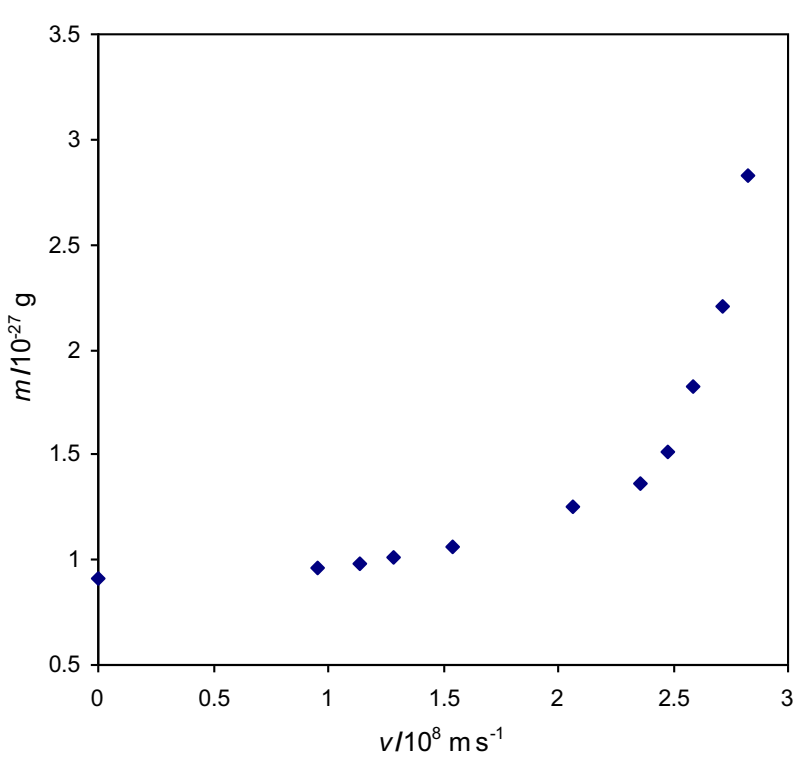

Table 1 Experimental values of $\alpha$ and $\beta$ (Shankland 1960), and values of $\alpha$ for the simple functions in the text (deviations in brackets)

\begin{tabular}{llllll}
\hline$\beta$ & $\alpha$ & $\alpha_{1}$ & $\alpha_{2}$ & $\alpha_{3}$ & $\alpha_{4}$ \\
\hline 0.317 & 1.06 & $1.46(+0.40)$ & $1.11(+0.05)$ & $1.21(+0.15)$ & $1.05(-0.01)$ \\
0.379 & 1.08 & $1.61(+0.53)$ & $1.17(+0.09)$ & $1.27(+0.19)$ & $1.08(0.00)$ \\
0.428 & 1.11 & $1.75(+0.64)$ & $1.22(+0.11)$ & $1.32(+0.21)$ & $1.11(0.00)$ \\
0.515 & 1.16 & $2.06(+0.90)$ & $1.36(+0.20)$ & $1.44(+0.28)$ & $1.17(+0.01)$ \\
0.687 & 1.37 & $3.19(+1.82)$ & $1.89(+0.52)$ & $1.79(+0.42)$ & $1.38(+0.01)$ \\
0.786 & 1.50 & $4.67(+3.17)$ & $2.62(+1.12)$ & $2.16(+0.66)$ & $1.62(+0.12)$ \\
0.826 & 1.66 & $5.75(+4.09)$ & $3.15(+1.49)$ & $2.40(+0.74)$ & $1.77(+0.11)$ \\
0.863 & 2.00 & $7.30(+5.30)$ & $3.92(+1.92)$ & $2.70(+0.70)$ & $1.98(-0.02)$ \\
0.906 & 2.42 & $10.64(+8.22)$ & $5.58(+3.16)$ & $3.26(+0.84)$ & $2.36(-0.06)$ \\
0.943 & 3.10 & $17.54(+14.44)$ & $9.03(+5.93)$ & $4.19(+1.09)$ & $3.00(-0.10)$
\end{tabular}

\section{Relation between mass and energy}

Equation 2 follows from Eq. 1. The latter can be differentiated by first taking logarithms. The result is

$$
\mathrm{d} m / m=v \mathrm{~d} v /\left(c^{2}-v^{2}\right)
$$

Rearrangement of this gives

$$
c^{2} \mathrm{~d} m=m v \mathrm{~d} v+v^{2} \mathrm{~d} m=v \mathrm{~d}(m v)=v \mathrm{~d} p
$$

where $p$ is the momentum. This can be written in terms of force (= rate of change of momentum) and energy ( $=$ force $\times$ distance): 


$$
c^{2} \mathrm{~d} m=(\mathrm{d} x / \mathrm{d} t) \mathrm{d} p=(\mathrm{d} p / \mathrm{d} t) \mathrm{d} x=F \mathrm{~d} x=\mathrm{d} E
$$

Integration from $E=0$ at $m=0$ gives Eq. 2 .

Equation 2 can be tested experimentally by measuring the energies of nuclear processes. Oliphant et al. (1935) studied the bombardment of lithium with protons to give $\alpha$-particles:

$$
{ }^{7} \mathrm{Li}+{ }^{1} \mathrm{H} \rightarrow{ }^{4} \mathrm{He}+{ }^{4} \mathrm{He}
$$

They found the energy of this to be $1.706 \times 10^{7} \mathrm{eV}=2.733 \times 10^{-12} \mathrm{~J}$. From Eq. 2, this represents a loss in mass of $3.041 \times 10^{-26} \mathrm{~g}$ or $0.01831 \mathrm{u}$. Mass spectrometry gives 0.01862 u (Cohen et al. 2007).

Physicists have recently made a much more precise test of Eq. 2 by comparing the reduction in total mass when an atom captures a neutron with the energy $(h v)$ of the $\gamma$ rays emitted (Rainville et al. 2005; Jentschel et al. 2009). Because the mass of a neutron is difficult to measure accurately without using Eq. 2, it is necessary to compare the results for two processes, e.g. ${ }^{1} \mathrm{H}+\mathrm{n} \rightarrow{ }^{2} \mathrm{H}$ and ${ }^{28} \mathrm{Si}+\mathrm{n} \rightarrow{ }^{29} \mathrm{Si}$. Results for ${ }^{1} \mathrm{H} /{ }^{28} \mathrm{Si}$ and ${ }^{1} \mathrm{H} /{ }^{32} \mathrm{~S}$ indicate that Eq. 2 holds to better than $\pm 0.00005 \%$.

\section{Discussion}

The approach I have described has the merit of tying Eqs. 1 and 2 closely to experiment. This takes away some of the mystery. If they are presented without proof, they are very puzzling. Interested students can go on to study Einstein's derivation of these equations (Miller 1997) later.

A second merit of my approach is that it invites the question of whether there is a better way of understanding the experimental data than on the theory of relativity. It is certainly not obvious that we should interpret the cathode-ray results by imagining two observers, one alongside the beam and another travelling with it. A more obvious explanation is that the particles are moving in a sub-electronic medium that retards their motion as their speed approaches $c$, much as a viscous liquid restricts the speed of particles moving through it to a limiting value. This was how physicists understood the phenomenon before Einstein (Miller 1997). Indeed, Lorentz obtained equations that are mathematically equivalent to Einstein's by thinking of the phenomenon in this way. While almost all physicists today accept the theory of relativity, there are some who question it, and the interpretation of the Michelson-Morley experiment on which it is based (Dunning-Davies 2007: Chap. 2).

\section{Reformulation}

This discussion supports the reformulation of Eqs. 1 and 2 in terms of constant mass (Kolbenstvedt and Stølevik 1991). If $m$ is taken to represent apparent mass ( $\left.m_{\text {app. }}\right)$ and $m_{0}$ actual mass $\left(m_{\text {act. }}\right)$, substitution of Eq. 1 into Eq. 2 gives

$$
E=E_{0}\left(1-v^{2} / c^{2}\right)^{-1 / 2}
$$

with

$$
E_{0}=m_{(\text {act. })} c^{2}
$$

For further discussion, see Kolbenstvedt and Stølevik (1991). 
Open Access This article is distributed under the terms of the Creative Commons Attribution 4.0 International License (http://creativecommons.org/licenses/by/4.0/), which permits unrestricted use, distribution, and reproduction in any medium, provided you give appropriate credit to the original author(s) and the source, provide a link to the Creative Commons license, and indicate if changes were made.

\section{References}

Atwood, C.H., Sheline, R.K.: J. Chem. Educ. 66, 389 (1989)

Balasubramanian, K.: Relativistic effects in chemistry, vols. 1 and 2. Wiley, New York (1997)

Banna, M.S.: J. Chem. Educ. 62, 197 (1985)

Bucherer, A.H.: Ann. Phys. 28, 513 (1909)

Cohen, E.R., et al.: Quantities, units and symbols in physical chemistry, 3rd edn. IUPAC, RSC, Cambridge (2007)

Dunning-Davies, J.: Exploding a myth. Horwood, Chichester (2007)

Grimm, H.G., Sommerfeld, A.: Z. Phys. 36, 36 (1926)

Guye, C.E., Lavanchy, C.: Compt. Rend. 161, 52 (1915)

Hopkins, K.G.G., Nelson, P.G.: J. Chem. Soc. Dalton Trans. 1393 (1984)

Jentschel, M., Krempel, J., Mutti, P.: Eur. Phys. J. Spec. Top. 172, 353 (2009)

Kaufmann, W.: Gött. Nach 2, 143 (1901)

Kolbenstvedt, H., Stølevik, R.: J. Chem. Educ. 68, 826 (1991)

Miller, A.I.: Albert Einstein's special theory of relativity: emergence (1905) and early interpretation (1905 - 1911). Springer, New York (1997)

Nelson, P.G.: Chem. Educ. Res. Pract. 3, 215 (2002)

Norrby, L.J.: J. Chem. Educ. 68, 110 (1991)

Oliphant, M.L.E., Kempton, A.R., Rutherford, Ld.: Proc. R. Soc. A 149, 406 (1935)

Rainville, S., et al.: Nature 438, 1096 (2005)

Rogers, M.M., McReynolds, A.W., Rogers Jr., F.T.: Phys. Rev. 57, 379 (1940)

Shankland, R.S.: Atomic and nuclear physics, 2nd edn. Macmillan, New York (1960)

Sidgwick, N.V.: The electronic theory of valency. Oxford University Press, Oxford (1927)

Thayer, J.S.: J. Chem. Educ. 82, 1721 (2005) 